Research Article

\title{
Nonlinear Noise Compensation in an Integration System of Fiber-Wireless Communication and Free Space Optical Communication
}

\author{
Long Chen $\mathbb{D}^{D}$, Miao Liu, and Zhijun Tang \\ School of Information and Electrical Engineering, Hunan University of Science and Technology, Xiangtan 411201, China \\ Correspondence should be addressed to Long Chen; cl-th@163.com
}

Received 13 August 2020; Revised 13 September 2020; Accepted 22 September 2020; Published 6 October 2020

Academic Editor: Junmin Liu

Copyright (c) 2020 Long Chen et al. This is an open access article distributed under the Creative Commons Attribution License, which permits unrestricted use, distribution, and reproduction in any medium, provided the original work is properly cited.

\begin{abstract}
We propose and experiment an integration system of fiber-wireless communication and free space optical (FSO) communication based on nonlinear noise compensation. This integration system, combining the benefits of FSO communication and highfrequency wireless communication, as well as the high-frequency efficiency and anti-interference to multipath fading of the multicarriers orthogonal frequency division multiplexing (OFDM) modulation, can increase the capacity and flexibility of the system. The nonlinear equalizer is employed to compensate the distortion caused by the beating between OFDM signal subcarriers in the combination system. Up to 1-Gbaud 16QAM discrete multitone (DMT) OFDM signal can be first transmitted over the distance of 100-m single-mode fiber (SMF), then delivered over the distance of 100-m FSO link, and finally, transmitted over the distance of $4 \mathrm{~m}$ wireless. The bit-error-ratio (BER) of the OFDM signal in the integration system can be less than the hard-decision forward-error-correction (HD-FEC) threshold of $3.8 \times 10^{-3}$. The experiment shows that the nonlinear equalizer can work well compatible with the combination system and can improve the transmission performance by $0.7-\mathrm{dB}$ receiving sensitivity in the combination system.
\end{abstract}

\section{Introduction}

Nowadays, wireless and broadband are becoming the main development trends of communication industry and even the whole information industry. The wireless transmission capacity will increase explosively, and the demand for ultra-high-speed wireless signal is imminent. The high-frequency millimeter wave, with higher frequency and large bandwidth, can increase data transmission rate and play an important role in achieving the requirements of Gbit/s wireless transmission [1-3]. Photon-assisted millimeter wave technology is an effective scheme of generating millimeter wave because it can overcome the limitation of the electronic devices bandwidth. Furthermore, photon-assisted millimeter wave technology has the characteristics of combining the optical communication and the wireless communication seamlessly. Fiber-wireless communication has attracted increasing interest by building an electronic system based on photonic technologies [4-6]. Free space optical communication, with the benefits of good beam directionality and low link power loss, is regarded as an effective access technology [7, 8]. The fiber-wireless communication and free space optical communication have different advantages. As compared with the FSO communication system, millimeter wave signal has stronger ability of passing through cloud penetration and diffracting the obstacles. As compared with the millimeter wave communication, the FSO communication system shows better security and electromagnetic interference immunity. The seamless combination of the two systems can enhance the reliability and flexibility of the transmission in the emergency services. The OFDM technique based on the high-order quadrature amplitude modulation (QAM), with the ability of resisting the influence caused by the multipath interference in wireless link and the ability of suppressing dispersion in fiber link, shows the characteristic of high-frequency efficiency and large 
transmission capacity in the communication system [9-11]. The OFDM modulation can be an effective modulation format for both the fiber-wireless communication system and the FSO communication. Based on the abovementioned factors, we have proposed a new integration system of the fiber-wireless communication and the FSO communication. The signal transmitted in the seamless combination system is used in the OFDM modulation [12]. However, there are linear and nonlinear effects in this integration system. As compared with the linear effects, the nonlinear effects will reduce the transmission performance of the integration system more seriously. There are many kinds of nonlinear effects in the integration system. These factors causing nonlinear effects include the working of optoelectronic components in the saturated region, the nonlinear effects in the optical fiber link, and the beating nonlinear noise produced by the square law detector at the receiver. In order to reduce the influence of the nonlinear effects in the integration system, we can reduce the transmitted power of the electrical signal and the launched power into optical fiber so that the devices and components in the system can work in their linear dynamic range. This method may reduce the performance of the system because of the low signal to noise ratio (SNR). Therefore, it is necessary to find an effective method to reduce the nonlinear effect. How to compensate nonlinear damage for the integration system is an important issue.

In this paper, we propose and experiment an integration system of the fiber-wireless communication and the FSO communication with nonlinear noise compensation. For the nonlinear OFDM subcarrier beating noise in the combination system, the Volterra nonlinear compensation equalizer is utilized to remove the noise. For the proposed system, the transmission signal is modulated to a light wave by direct modulation at the sending terminal, and the high-frequency millimeter wave is downconverted to a baseband signal by direct detection at the receiving terminal. Furthermore, an $\mathrm{H}$-averaging technique and spread spectrum based on discrete Fourier transform are utilized for the optimization of the transmission performance $[5,13]$. In the experiment, up to 1 Gbaud 16QAM-DMT signal can finish $100 \mathrm{~m}$ SMF and $100 \mathrm{~m}$ FSO link transmission and, then, achieve delivery of $4 \mathrm{~m}$ wireless link with a BER under $3.8 \times 10^{-3}$. The results reveal that the nonlinear noise compensation can optimize the transmission performance by $0.7 \mathrm{~dB}$ receiving sensitivity for eliminating the noise caused by the beating between DMT subcarriers.

1.1. Principle of the Integration System Based on Nonlinear Noise Compensation. Figure 1 depicts the structure of the integration system based on the nonlinear noise compensation. The two optical signals at the fiber transmission part of the integration system are used to generate millimeter wave over fiber. Only one optical signal carries the data. The electrical baseband OFDM signal is modulated to the optical signal with a central wavelength $\lambda_{1}$ by the directly modulated laser (DML). The other optical signal without data is a continuous light wave with a central wavelength $\lambda_{2}$, which is emitted by an adjustable laser and can adjust the difference between $\lambda_{2}$ and $\lambda_{1}$ according to the required frequency of the millimeter wave. The two waves are coupled in the optical coupler (OC) in Figure 1, and the spectral diagram of the signal after optical coupling at point a in Figure 1 is shown in Figure 2(a). The frequency interval between wavelength $\lambda_{1}$ and wavelength $\lambda_{2}$ is equal to the frequency $f_{\mathrm{RF}}$ of the produced high-frequency millimeter wave. The frequency components of the transmitted OFDM subcarriers are located at wavelength $\lambda_{1}$. The coupled optical signal carrying millimeter wave is amplified by the optical amplifier and transmitted into the SMF and, then, sent to the free space optical link. At the FSO link terminal, an optical antenna is used to receive the optical signal carrying millimeter wave. After that, the optical signal carrying millimeter wave completes the optical fiber link and the FSO link delivery. The spectral diagram of the received signal at point $b$ in Figure 1 is shown in Figure 2(b). The received optical signal, then, enters the photodetector to generate a high-frequency electrical millimeter wave. The spectrum diagram of the electrical millimeter wave at point $c$ in Figure 1 is shown in Figure 2(c). Figure 2(c) reveals that the signal after the PD not only carries the OFDM signal at the high-frequency millimeter wave but also produces the baseband OFDM signal with nonlinear noise. Because the square law detection of photoelectric detection will lead to the interference of subcarriers beating in the OFDM signal, nonlinear noise is generated at the baseband. The highfrequency millimeter wave signal is not disturbed by the subcarriers beating noise. Next, the millimeter wave signal is transmitted wirelessly through the antenna. The millimeter wave is captured by another antenna after the wireless transmission link, and the spectrum diagram of the signal at point $d$ in Figure 1 is shown in Figure 2(d). As compared with the signal in Figure 2(c), the signal in Figure 2(d) lacks the signal part at low frequency. The electrical signal transmitted from point $c$ to the point $d$ needs to pass through the devices working at the $\mathrm{W}$-band frequency range, resulting in the low-frequency part of the signal being filtered out. The envelope detection is adopted to realize the frequency downconversion of the OFDM signal. The spectrum diagram of the signal at point $e$ in Figure 1 is shown in Figure 2(e). We can see from Figure 2(e) that the baseband OFDM signal will carry nonlinear noise due to the subcarriers beating after the square law detection.

The nonlinear equalizer has a good effect in suppressing the nonlinear noise in the communication system, among which the Volterra nonlinear equalizer is a 


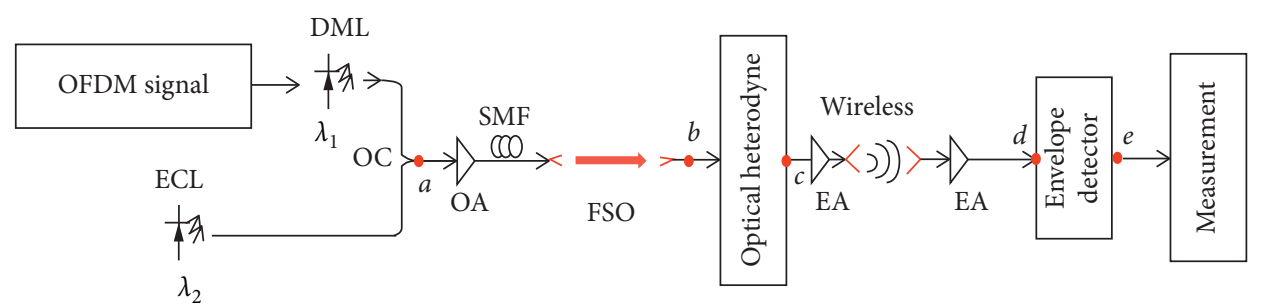

Figure 1: Structure of the integration System.
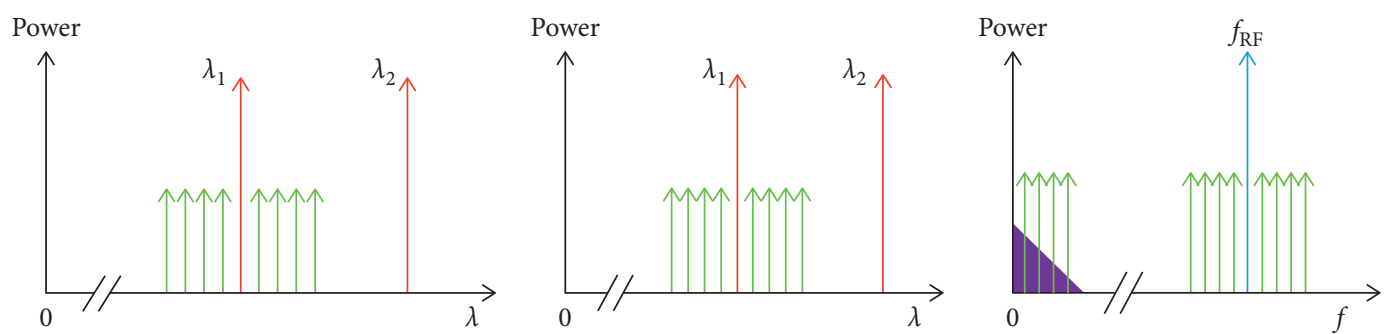

$\uparrow \uparrow \uparrow \uparrow$ OFDM subcarriers
$\mathbf{N}$ Nonlinear noise

$\uparrow \uparrow \uparrow \uparrow$ OFDM subcarriers

- Nonlinear noise

(b) $\uparrow \uparrow \uparrow \uparrow$ OFDM subcarriers

_ Nonlinear noise (a)

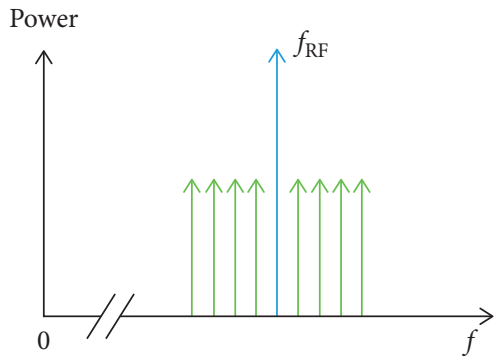

$\uparrow \uparrow \uparrow \uparrow$ OFDM subcarriers

- Nonlinear noise

(d)
Power

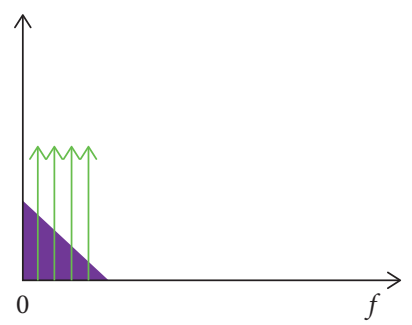

$\uparrow \uparrow \uparrow \uparrow$ OFDM subcarriers

- Nonlinear noise

(e)

Figure 2: Spectrum diagrams in the integration system. (a) After optical coupling. (b) After FSO link. (c) After the PD. (d) After wireless transmission. (e) After envelope detection.

good choice with development potential [14]. The mathematical expression of the adaptive Volterra nonlinear equalizer is

$$
\begin{aligned}
y(t)= & w_{0}+\sum_{k=0}^{M-1} w_{1}(k) x(t-k)+\sum_{k_{1}=0}^{M-1} \sum_{k_{2}=k_{1}}^{M-1} \\
& \cdot w_{2}\left(k_{1}, k_{2}\right) x\left(t-k_{1}\right) x\left(t-k_{2}\right) \\
& +\cdots+\sum_{k_{1}=0}^{M-1} \cdots \sum_{k_{n}=k_{n-1}}^{M-1} \\
& \cdot w_{n}\left(k_{1}, \ldots, k_{n}\right) x\left(t-k_{1}\right) \cdots x\left(t-k_{n}\right),
\end{aligned}
$$

where $\left(w_{0}, w_{1}, w_{2}, \ldots, w_{n}\right)$ represent the parameters of the equalizer, $x(t)$ stands for the input of the system, and $y(t)$ stands for the output after the Volterra equalizer. Due to the contradiction between the computational complexity of algorithm and the counting accuracy of the Volterra filter, the implementation process can reserve the first- and the second-order terms and discard the higher-order terms. In the communication systems, the signal with OFDM modulation usually produces nonlinear noise because of subcarrier beating after the square law envelope detector. Reference [14] proposed a nonlinear equalizer for discrete multitone OFDM modulated signals for compensation of both linear and nonlinear noises.

The Volterra equalizer consists of two finite impulse response filters. One adaptive filter is used for the compensation of linear distortion. Another one is designed for the compensation of nonlinear distortion. The mathematical formula for the Volterra equalizer in reference [14] is given as

$$
y_{i}=\sum_{j=-M}^{M} c_{j} x_{i-j}+\sum_{l=-M}^{M} \sum_{j=-M}^{l-1} h_{l, j}\left(x_{i-l}-x_{i-j}\right)^{2} \text {, }
$$

where $c_{j}$ denote the weight parameters of the linear filter in the first-order terms, and $h_{l, j}$ denote the weight parameters 
of the nonlinear filter in the second-order terms. $M$ stands for the number of the taps in two filters.

The Volterra equalizer shown in equation (2) is applied to the integration system to eliminate nonlinear noise. By using the least mean squares (LMS) algorithm, we obtain the weight parameters of the first- and the second-order terms. Combined with the OFDM signal transmitted in the proposed combination system, the calculating formulas used in this system are given by

$$
\begin{aligned}
\varepsilon_{i} & =d_{i}-y_{i}, \\
c_{j} & =c_{j}+\mu_{c} \varepsilon_{i} x_{i} \quad j=-M,-M+1, \cdots, M, \\
h_{l, j} & =h_{l, j}+\mu_{h} \varepsilon_{i}\left(x_{i-l}-x_{i-j}\right)^{2}, \\
j & =-M,-M+1, \cdots, l-1 ; l=-M,-M+1, \cdots, M,
\end{aligned}
$$

where $\mu_{c}$ stands for the step coefficients in the linear filter, $\mu_{h}$ stands for the step coefficients in the nonlinear filter, and $\varepsilon_{i}$ denotes the difference between the actual values and the theoretical values.

\section{Experimental Setup}

Figure 3 depicts the experimental diagram of the combination communication system based on the nonlinear noise compensation. At first, the OFDM modulation is finished in offline Matlab software. Among OFDM signal modulation, the subcarriers number used to transmit the data is 100, and these subcarriers use 16QAM high-order vector mapping to achieve high spectral efficiency of the signal. The spread spectrum technology based on discrete Fourier transform is used in the system. This technology requires an additional FFT on the modulation end and an additional IFFT on the demodulation end on the basis of traditional OFDM modulation. The 100 subcarriers are first FFT transformed and, then, the 100 data-carrying subcarriers of different frequencies are processed by conjugate symmetric operation so that the 100 subcarriers are converted to 200 subcarriers of different frequencies for realizing discrete multitone-type OFDM signal modulation, as shown in the Figure 3. The traditional OFDM signals are complex values, while the discrete multitone-type OFDM signals become real values with the additional conjugate symmetric operation. In order to prevent interference between subcarriers, a DC component 0 in the first position and 24 protection intervals are added to the converted subcarriers satisfying conjugate symmetry. Then, all the subcarriers obtained are carried out with the IFFT transformation. Finally, a cyclic prefix of 0.125 is added to the data after the IFFT transformation. Based on the abovementioned operations, one symbol of the discrete multitone OFDM signal is modulated. A training sequence, with the same length to one OFDM symbol, is inserted to the OFDM symbols before the signal complete modulation offline. The electrical 1-Gbaud 16QAM-DMT-OFDM signal, produced by an arbitrary waveform generator (AWG), is, then, modulated to the optical signal by a DML. As one of the coupled lights, the center wavelength of the optical signal output from the DML is $159.9995 \mathrm{~nm}$ and the output optical power of the DML is $2.26 \mathrm{~mW}$. The other light wave is produced by an external cavity laser (ECL) with the center wavelength controlled to be $1549.386 \mathrm{~nm}$. After coupling two optical signals, the frequency interval between the center carriers of the two optical signal is $75 \mathrm{GHz}$. Then, the optical signal carrying millimeter wave signal is amplified to $13 \mathrm{dBm}$ through an erbium-doped fiber amplifier and transmitted into optical fiber for $100 \mathrm{~m}$ transmission. Next, the amplified optical signal carrying the millimeter wave signal is transmitted by an optical antenna through a $100 \mathrm{~m}$ FSO link. Then, the optical signal with millimeter wave is captured by another optical antenna as shown in Figure 3.

After free space optical transmission, the power of the optical signal carrying millimeter wave before the photoelectric conversion is maintained to be the appropriate value through an EDFA and an optical attenuator. Subsequently, the optical signal with the millimeter wave realizes the photoelectric conversion to be an electrical signal at $75 \mathrm{GHz}$ through a photoelectric detector with $3 \mathrm{~dB}$ bandwidth of $100 \mathrm{GHz}$ for realizing the $\mathrm{O} / \mathrm{E}$ conversion. The generated high-frequency millimeter wave at $75 \mathrm{GHz}$ is amplified by an amplifier with $4-\mathrm{dBm}$ saturated output power and $32-\mathrm{dB}$ gain. The electrical amplifier works at the frequency range of $\mathrm{W}$-band. Then, the electric high-frequency millimeter wave signal is emitted by a Cassegrain antenna with a gain of 50.8$\mathrm{dBi}$ and a transmitting beam width of 0.4 degrees. After transmitting a wireless distance of $4 \mathrm{~m}$, the millimeter wave was captured by another Cassegrain antenna with the same parameters as the one on the wireless transmitting end. Then, the electrical OFDM signal at $75 \mathrm{GHz}$ is amplified by another electrical amplifier which is the same as the one on the wireless transmitting end. Then, the OFDM signal at $75 \mathrm{GHz}$ is downconverted into a baseband electrical OFDM signal by the envelope detector. Then, the baseband DMT-OFDM signal is amplified by an electrical amplifier with a working frequency of $0 \sim 40 \mathrm{GHz}$. Next, a digital oscilloscope, with 50$\mathrm{GSa} / \mathrm{s}$ sampling rate and $16 \mathrm{GHz}$ bandwidth, is used to capture the electrical baseband 16QAM-DMT-OFDM signal. Finally, the 16QAM-DMT-OFDM signal demodulation and the BER counting are carried out in Matlab software. As shown in the Figure 3, the demodulation includes synchronization, CP removing, FFT, guard removing, IFFT, and symbol demapping. As compared with the modulation, the demodulation has the opposite processes. During demodulation, we use the proposed LMS-based Volterra equalizer to compensate the nonlinear noise. Moreover, the H-averaging technique with 7 taps and spread spectrum technique based on discrete Fourier transform are employed in the integration system to improve the transmission performance.

\section{Experimental Results}

Figures 4(a) and 4(b) show the electrical spectrums of the OFDM signal transmitted after the AWG and the OFDM signal received in the OSC, respectively. The signal 


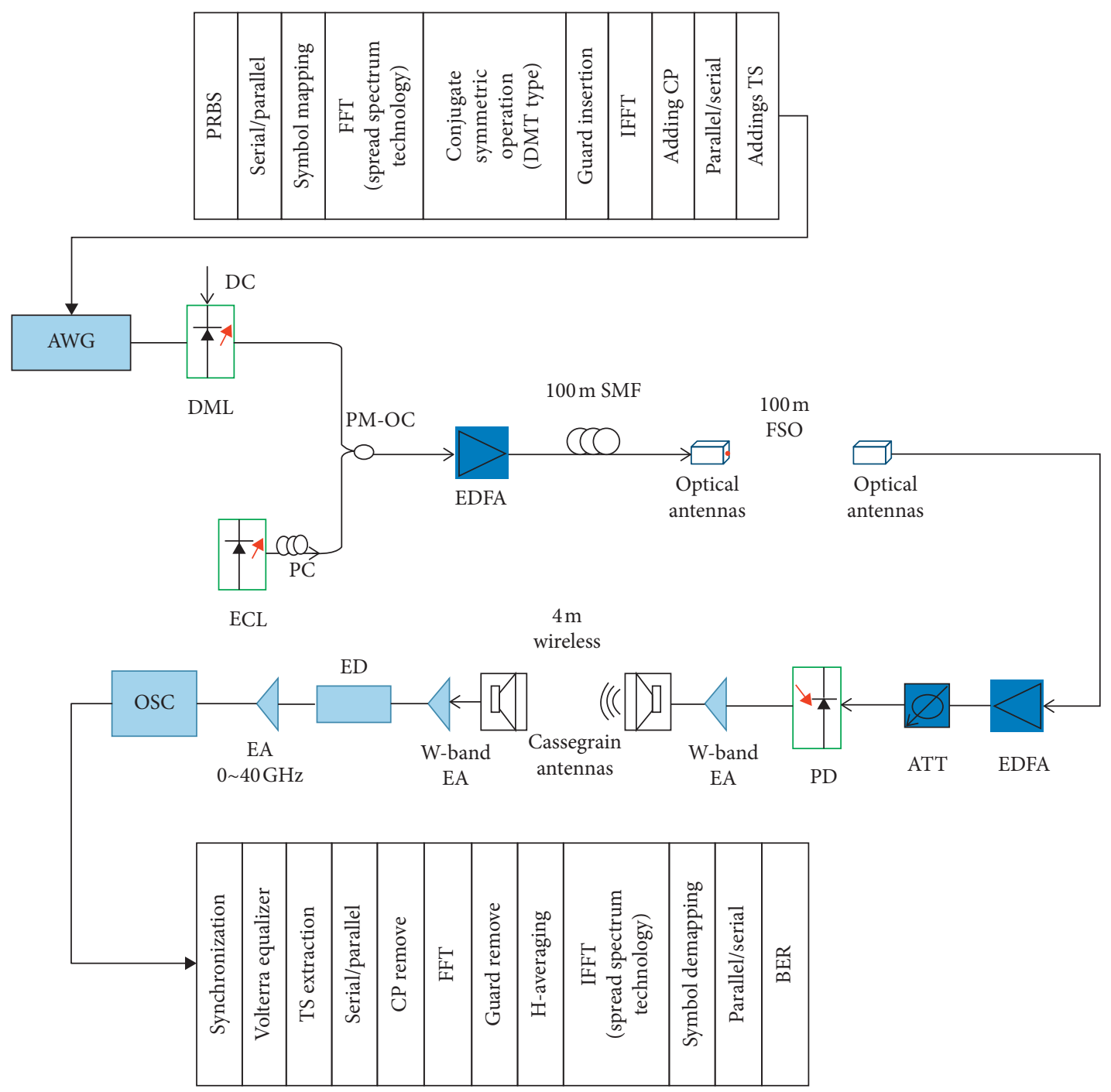

FIgURE 3: Experimental setup diagram of the fusion system.

transmission rate in the experiment is $1-G b a u d$. From the spectrums, it can be seen that the SNR of the signal decreases seriously after the optical channel and the wireless channel. The high-frequency parts of the OFDM signal attenuate more seriously because of the frequency-selective fading in the devices and link channel. In addition, as compared with the transmitted signal, the received signal is also affected by the interference of subcarriers beating described above. Also, the subcarriers at lower frequency are relatively affected more serious. Figure 4(c) depicts the optical spectrum of the coupled optical signal carrying millimeter wave. The difference between the continuous optical wavelength $\lambda_{2}$ and $\lambda_{1}$ can be adjusted according to the required high frequency of the millimeter wave. In the experiment, we set the frequency difference between the central carriers to be $75 \mathrm{GHz}$, so that the $75 \mathrm{GHz}$ millimeter wave is generated in the system.

Figure 5(a) shows the constellation of the 1-Gbaud OFDM signal. In this case, the nonlinear equalizer is not used in the integration system, and the launched power of the signal into $\mathrm{PD}$ is $-0.5 \mathrm{dBm}$. Figure $5(\mathrm{~b})$ shows the constellation of the 1-Gbaud OFDM signal. In this case, the nonlinear equalizer is employed in the integration system, and the launched power of the signal into $\mathrm{PD}$ is also $-0.5 \mathrm{dBm}$. The results reveal that the constellation of the latter has obviously more convergence than that of the former with the same transmission rate.

Figure 6 depicts the measured BER curves of the transmission OFDM signal with the nonlinear equalizer and without the nonlinear equalizer at the receiver. The transmission rate is 1-Gbaud in each case. It can be seen from the result that the system performance with the nonlinear equalizer is better under the same rate. By compensating the nonlinear noise, the Volterra equalizer can optimize the performance of the combination system and improve the receiving sensitivity by $0.7 \mathrm{~dB}$. When the launched power of the signal into PD is more than $-0.5 \mathrm{dBm}$, the BER of the OFDM signal transmitted in this combination system with the nonlinear equalizer compensation can be less than $3.8 \times 10^{-3}$. 


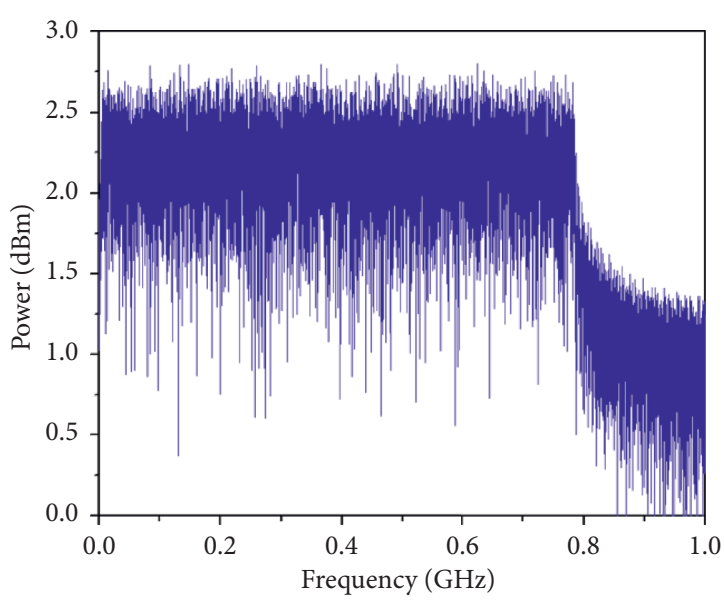

(a)



(b)

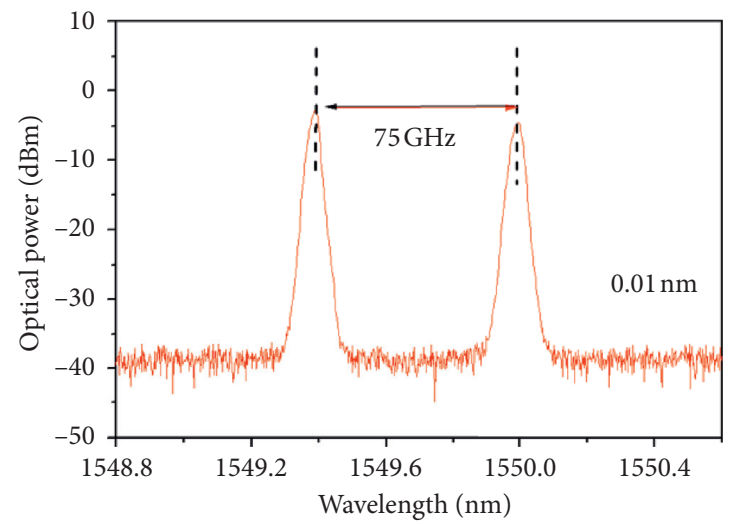

(c)

FIgURE 4: Experimental spectrums. (a) The OFDM signal transmitted after the AWG. (b) The OFDM signal received in the OSC. (c) The coupled optical signal carrying millimeter wave.

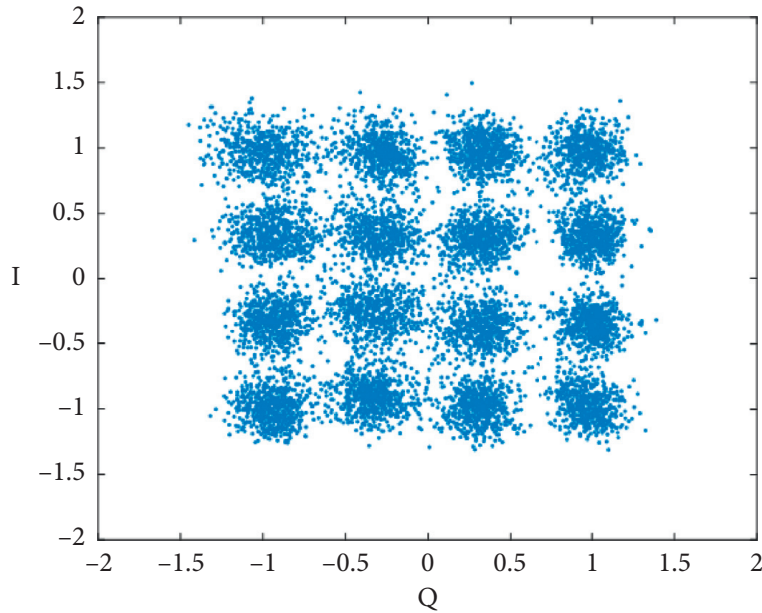

(a)

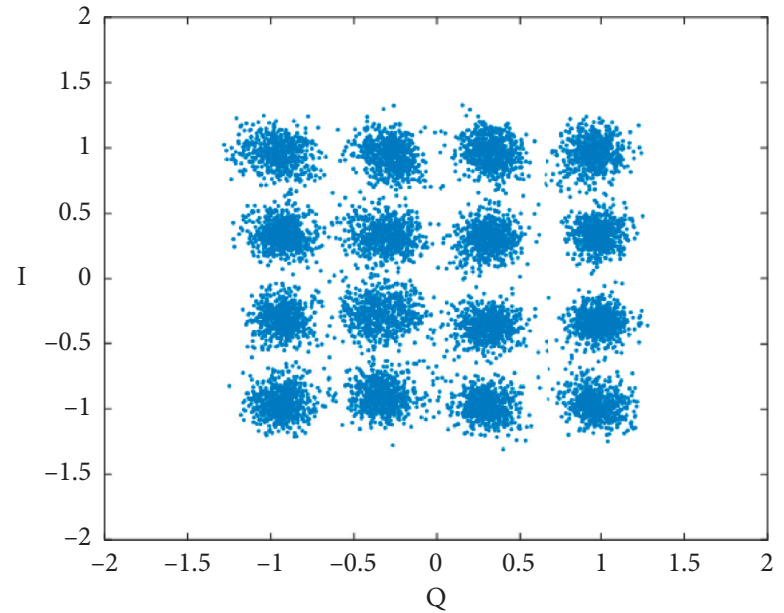

(b)

FIgURE 5: Received constellation. 


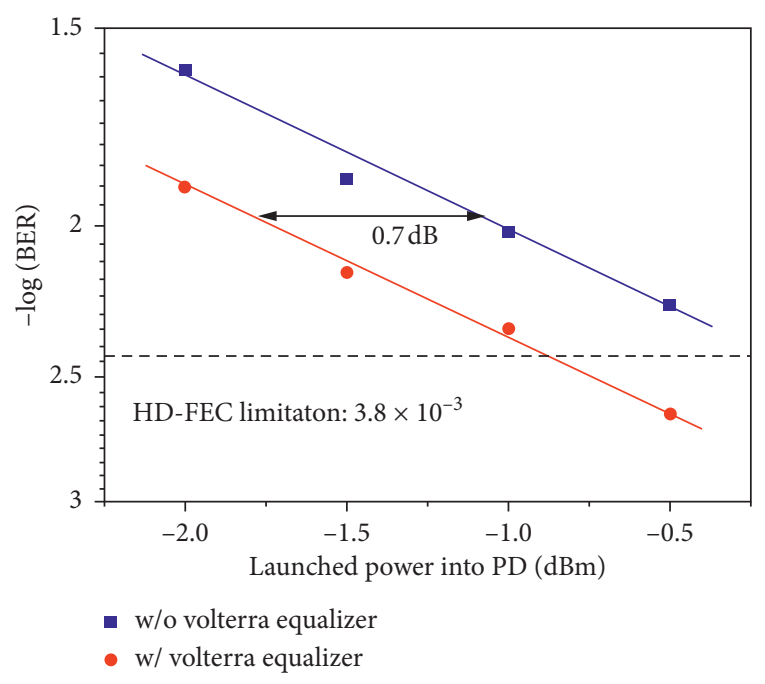

FIGURE 6: BER curves of the OFDM signal with and without the Volterra nonlinear equalizer.

\section{Conclusions}

We propose and experiment an integration system of fiberwireless communication and FSO communication based on nonlinear noise compensation. This integration system, combining the benefits of FSO communication and highfrequency wireless communication, as well as the highfrequency efficiency and anti-interference to multipath fading of the multicarriers OFDM modulation, can increase the capacity and flexibility of the system. The nonlinear equalizer is employed to compensate the distortion caused by the beating between OFDM signal subcarriers in the combination system. Up to 1-Gbaud 16QAM-DMT- OFDM signal can be first transmitted over the distance of $100-\mathrm{m}$ SMF, then delivered over the distance of 100-m FSO link, and finally, transmitted over the distance of $4 \mathrm{~m}$ wireless. The BER of the OFDM signal in the integration system can be less than the HD-FEC threshold of $3.8 \times 10^{-3}$. The experiment shows that the nonlinear equalizer can work well compatible with the combination system and can improve the transmission performance by $0.7-\mathrm{dB}$ receiving sensitivity in the combination system.

\section{Data Availability}

All data used to support the findings of this study are available from the corresponding author upon request.

\section{Conflicts of Interest}

The authors declare that there are no conflict of interest regarding the publication of this paper.

\section{Acknowledgments}

This paper was supported by the National Natural Science Foundation of China (Grant nos. 61905074 and 61875054); the Hunan Provincial Natural Science Foundation of China (Grant nos. 2019JJ50170, 2020JJ7065 and 2020JJ4318); and the Scientific Research Fund of Hunan Provincial Education Department (18C0326).

\section{References}

[1] R. W. Heath, N. Gonzalez-Prelcic, S. Rangan, W. Roh, and A. M. Sayeed, "An overview of signal processing techniques for millimeter wave MIMO systems," IEEE Journal of Selected Topics in Signal Processing, vol. 10, no. 3, pp. 436-453, 2016.

[2] J. Xiao, X. Li, Y. Xu, Z. Zhang, L. Chen, and J. Yu, "W-band OFDM photonic vector signal generation employing a single Mach-Zehnder modulator and precoding," Optics Express, vol. 23, no. 18, pp. 24029-24034, 2015.

[3] H. N. Parajuli, H. Shams, L. G. Gonzalez, E. Udvary, C. Renaud, and J. Mitchell, "Experimental demonstration of multi-gbps multi sub-bands FBMC transmission in mm-wave radio over a fiber system," Optics Express, vol. 26, no. 6, pp. 7306-7312, 2018.

[4] J. L. Li, F. Zhao, and J. Yu, "D-band millimeter wave generation and transmission though radio-over-fiber system," IEEE Photonics Journal, vol. 12, no. 2, p. 5500708, 2020.

[5] L. Chen, Z. W. He, L. Zhao et al., "60 GHz reverse modulation fiber-wireless system with two jointed DSP algorithms," Advances in Condensed Matter Physics, vol. 2018, Article ID 7256162, 6 pages, 2018.

[6] X. Li, J. Yu, and G.-K. Chang, "Photonics-assisted technologies for extreme broadband $5 \mathrm{G}$ wireless communications," Journal of Lightwave Technology, vol. 37, no. 12, pp. 28512865, 2019.

[7] J. C. Juarez, A. Dwivedi, A. R. Hammons, S. D. Jones, V. Weerackody, and R. A. Nichols, "Free-space optical communications for next-generation military networks," IEEE Communications Magazine, vol. 44, no. 11, pp. 46-51, 2006.

[8] W. Liu, K. Yao, D. Huang, X. Lin, L. Wang, and Y. Lv, "Performance evaluation of coherent free space optical communications with a double-stage fast-steering-mirror adaptive optics system depending on the Greenwood frequency," Optics Express, vol. 24, no. 12, pp. 13288-13302, 2016.

[9] M. Chen, J. Yu, and X. Xiao, "Real-time Q-band OFDM-RoF systems with optical heterodyning and envelope detection for downlink transmission," IEEE Photonics Journal, vol. 9, no. 2, p. 7902007, 2017.

[10] X. Liu, P. Wang, T. Liu et al., “ABER performance of LDPCcoded OFDM free-space optical communication system over exponentiated weibull fading channels with pointing errors," IEEE Photonics Journal, vol. 9, no. 4, p. 7905113, 2017.

[11] S. E. Alavi, I. S. Amiri, M. Khalily et al., "W-band OFDM for radio-over-fiber direct-detection link enabled by frequency nonupling optical up-conversion," IEEE Photonics Journal, vol. 6, no. 6, p. 7903908, 2014.

[12] L. Chen, J. Luo, Z. Tang, and Z. Xi, "Integration system of photon-assisted $\mathrm{W}$-band millimeter wave wireless communication and free space optical communication based on OFDM signal transmission," Optical Fiber Technology, vol. 52, p. 101973, 2019.

[13] F. Li, X. Li, L. Chen et al., "High-level QAM OFDM system using DML for low-cost short reach optical communications," IEEE Photonics Technology Letters, vol. 26, no. 9, pp. 941-944, 2014.

[14] W. Yan, B. Liu, L. Li et al., "Nonlinear distortion and DSPbased compensation in metro and access networks using discrete multi-tone," in Proceedings of the Presented at the European Conference on Optical Communication, Amsterdam, The Netherlands, September 2012. 